\title{
Photometric and spectroscopic observations of (2060) Chiron at the ESO Very Large Telescope ${ }^{\star}$
}

\author{
J. Romon-Martin ${ }^{1}$, C. Delahodde ${ }^{2}$, M. A. Barucci ${ }^{1}$, C. de Bergh ${ }^{1}$, and N. Peixinho ${ }^{1,3}$ \\ ${ }^{1}$ Observatoire de Paris, Meudon, France \\ 2 Institut d'Astrophysique de Marseille, France \\ ${ }^{3}$ Centro de Astronomia e Astrofisica da Universidade de Lisboa, Portugal
}

Received 7 June 2002 / Accepted 9 December 2002

\begin{abstract}
Photometric and spectroscopic observations of Centaur Chiron have been obtained at the ESO/Very Large Telescope (VLT), both in optical and near-infrared spectral ranges, on 12-14 June 2001. Photometric optical data reveal that Chiron had reached a high activity level at that time. No absorption feature could be seen on the spectra; especially, no water ice could be detected.
\end{abstract}

Key words. comets: individual: Chiron - minor planets, asteroids - techniques: spectroscopic, photometric

\section{Introduction}

Centaur (2060) Chiron was discovered in 1977 (Kowal et al. 1977), and it was first classified as an asteroid. About ten years later, Tholen et al. (1988) found non-asteroidal brightness variations, and suggested that some kind of cometary activity may occur on Chiron. Other authors confirmed these changes in brightness (see e.g. Hartmann et al. 1990). In 1989, Meech \& Belton published the first direct detection of Chiron's coma. Other direct observations of the coma, as well as the detection of CN and CO emissions (Bus et al. 1991; Womack \& Stern 1995), confirmed the existence of cometary activity on Chiron. Thus Comet Chiron is also named 95/P Chiron.

Due to its brightness ( $m_{R} \simeq 16$ beyond $10 \mathrm{AU}$ ), Chiron is an ideal case to study distant small bodies. Chiron belongs to the Centaur population, which is probably a transiting population between the Kuiper Belt and the short-period comets (Levison \& Duncan 1997). Thus Chiron has been extensively studied since the end of the 1980s (see Barucci et al. 2002, for a complete review).

It is clearly established that Chiron's surface has neutral colors (see e.g. Hartmann et al. 1990). Three determinations of the albedo gave the following results: $p \simeq 0.12$ (Groussin et al. 2002), 0.17 (Fernández et al. 2002), and 0.14 (Campins et al. 1994). These values are consistent but may be affected by some dust scattering from the coma. The rotational period of

\footnotetext{
Send offprint requests to: J. Romon-Martin, e-mail: jennifer.romon@obspm.fr

* Based on observations made with ESO Telescopes at the Paranal Observatory (under programme ID 67.C-0171).
}

Chiron has been determined by several authors, who obtained values close to $\simeq 6 \mathrm{hrs}$. The variation in the amplitude of the lightcurve, ranging from 0.04 to 0.09 magnitude, is related to the cometary activity level (see e.g. Lazzaro et al. 1997). In the following, we will use the rotational period determined by Marcialis \& Buratti (1993): $P=5.917813 \pm 0.000007 \mathrm{hrs}$.

The near-infrared (NIR) spectra of Chiron that have been published until 1998 did not show any features. However, more recent near-infrared spectra (Foster et al. 1999, Luu et al. 2000) show a $2-\mu \mathrm{m}$ absorption band, indicating the presence of water ice on Chiron's surface.

In this paper, we present new photometric and spectroscopic data about Chiron, obtained with the 8-m Very Large Telescope (VLT). These observations are part of an observing programme focused on the study of variations of bright Centaur spectra over a rotational period. Our goal was also to confirm the detection of water ice on Chiron's surface.

\section{Observations and data reduction}

The observations were performed at the Very Large Telescope (European Southern Observatory, Chile) on 12-14 June 2001. At that time, 2060 Chiron was at a heliocentric distance of $10.76 \mathrm{AU}$, a geocentric distance of $9.75 \mathrm{AU}$, and a phase angle of $0.7^{\circ}$.

We obtained optical data using the FORS2 spectro-imager (on the 3rd Unit 8-m Telescope - UT3), and NIR data using the ISAAC spectro-imager (on UT1). Table 1 gives the journal of the observations. 
Table 1. Journal of the observations.

\begin{tabular}{cccc}
\hline \hline Time (UT) & Obs. mode & $\begin{array}{c}\text { Spectral } \\
\text { range }\end{array}$ & $\begin{array}{c}\text { Rot. phase } \\
\text { at exp. start }\end{array}$ \\
\hline 13 June 02:35 & Imaging & $J H K \mathrm{~s}$ & 0.02 \\
13 June 06:00 & Imaging & $B V R I$ & 0.60 \\
13 June 06:14 & Imaging & $J H K \mathrm{~s}$ & 0.64 \\
13 June 07:08 & Imaging & $B V R I$ & 0.79 \\
13 June 08:46 & Imaging & $B V R I$ & 0.07 \\
14 June 02:38 & Imaging & $J H K \mathrm{~s}$ & 0.09 \\
13 June 03:15 & Spectro. & $J S H S K$ & 0.13 \\
13 June 06:15 & Spectro. & $400-900 \mathrm{~nm}$ & 0.64 \\
13 June 07:28 & Spectro. & $400-900 \mathrm{~nm}$ & 0.85 \\
14 June 03:06 & Spectro. & $J S H S K$ & 0.17 \\
14 June 05:54 & Spectro. & $J S H S K$ & 0.64 \\
\hline
\end{tabular}

The zero point of the rotational phase is the same as used by Marcialis \& Buratti (1993).

To perform the photometric measurements, FORS2 was set in its direct imaging mode, with a pixel scale of $0.2^{\prime \prime}$ and a field of view of $6.8^{\prime} \times 6.8^{\prime}$. We used $B, V, R$, and $I$ filters, respectively centred at $429,554,655$, and $768 \mathrm{~nm}$. For each filter, the exposure time is $2 \mathrm{~s}$. FORS contains a rotating half-segment exposure shutter which guarantees uniform illumination of the CCD to the $1 \%$ level or better for exposure times as short as 1 second. Three $V-B-V-R-V-I-V$ sequences were performed. For the $J H K$ s imaging, ISAAC was set in its short wavelength $(\mathrm{SW})$ mode, which is equipped with a $1024 \times 1024$ $\mathrm{Hg}$ :Cd:Te array (field of view of $2.5^{\prime} \times 2.5^{\prime}$ ). We used the $J, H$, and $K$ s filters (centred at $1.25,1.65$, and $2.16 \mu \mathrm{m}$, respectively). The exposure time is 6 min per filter (six frames of 1 min each).

We have also obtained two optical spectra, using FORS2 with Grism 150I, and three NIR spectra, using ISAAC in its low resolution mode, with $J, S H$, and $S K$ filters. The optical spectra cover approximately the range $400-900 \mathrm{~nm}$. The NIR spectra cover the ranges $1.1-1.4 \mu \mathrm{m}(J), 1.45-1.8 \mu \mathrm{m}(S H)$, and $1.9-2.5 \mu \mathrm{m}(S K)$. Each band was obtained separately. The exposure times were $5 \mathrm{~min}$ in the optical range, $8 \mathrm{~min}$ in $J$ band, $12 \mathrm{~min}$ in $S H$ band, and $28 \mathrm{~min}$ in $S K$ band. The slit was $1^{\prime \prime}$ wide in both cases, providing a spectral resolution of about 200 in the optical range, and 500 in the NIR range. The slit is oriented along the object's motion in order to correct for any bad tracking.

Both imaging and spectroscopic frames have been corrected for bias and flat-field. Photometric measurements have been made using the MIDAS and ECLIPSE softwares. Photometric calibration of the optical data has been performed using 26 standard stars from the Landolt catalog (Landolt 1992), observed at different airmasses. Extinction coefficients, color terms and zero-points have been computed for each filter using the PHOTCAL package provided in the IRAF software. To perform the photometric calibration of the IR data, we have observed five stars from the HST/Nicmos list of faint IR standard stars.
After bias and flat-field correction, spectra were extracted from the frame using the TWODSPEC/APEXTRACT package provided in the IRAF software. The ECLIPSE software has been also used for IR spectroscopic data reduction, in addition to IRAF. The observation technique used for IR spectroscopy combines nodding along the slit and jittering to remove bad pixels. A complete description of the observation and data reduction technique can be found in Romon et al. (2001). Wavelength calibration has been performed using helium, $\mathrm{HgCd}$, and argon lamp spectra. Finally, Chiron's spectra have been corrected for the telluric and solar contributions, using solar analog spectra: optical spectra have been corrected using G3V-star HD 1835, and IR spectra have been corrected using C-type asteroids.

\section{Results}

\subsection{Photometric measurements}

$B V R I$ and $J H K$ magnitudes of Chiron have been measured from each imaging frame. Results are given in Table 2. Due to strong field crowding, aperture correction has been applied to most images (see Barucci et al. 2000), so that the measured magnitudes do not account for the total flux of Chiron. All the results shown in Table 2 were obtained using an aperture size of $1.4^{\prime \prime}$. For a few $V$ images, measurements of the magnitude corresponding to the total flux of Chiron could be performed, giving an estimate of the loss flux amounting to about $10 \%$ of the total flux.

No significant changes could be found in the magnitude measurements. Our data follow the general behaviour of the lightcurve published by Marcialis \& Buratti (1993).

On 13 June 2001, the mean apparent magnitude was $m_{V}=$ $16.10 \pm 0.02$, corresponding to an absolute magnitude $H_{V}=$ $5.95 \pm 0.02$, using $g=0.70$ as determined by Bus et al. (1989). The phase angle effects can be neglected since the phase angle was very small $\left(0.7^{\circ}\right)$ at the time of our observations. The determination of $H_{V}$ gives an indication of Chiron's activity level. Chiron's activity reached a maximum in October 1988 $\left(H_{V}=5.64\right.$, Hartmann et al. 1990). Its activity level has decreased since 1988, to reach $H_{V}=6.74$ in 1997 (Davies et al. 1998). On 13 June 2001, Chiron was nearly as bright as it was in 1988. A strong activity outburst would thus have occurred on Chiron in June 2001. Bauer et al. (2001) have also performed observations of Chiron in June 2001, finding $H_{V}=5.63 \pm 0.05$.

\subsection{Surface brightness profile}

In order to confirm the highly active state of Chiron, we have computed surface brightness profiles. For each of the three $V-B-V-R-V-I-V$ imaging sequences, we have obtained four $V$ frames that were averaged with respect to the apparent motion of the object. The three resulting images will be referred as $V_{1}, V_{2}$, and $V_{3}$. Using the $V_{1}, V_{2}$, and $V_{3}$ frames, we obtained surface brightness profiles of Chiron. We compared Chiron's profile to an average stellar profile. This stellar profile is the average profile of about 40 stars. Chiron's profile clearly 
Table 2. Optical and near-infrared photometric measurements.

\begin{tabular}{ccccc|cccc}
\hline \hline Time (UT) & $V$ mag. & $B-V$ & $V-R$ & $V-I$ & Time (UT) & $J$ mag. & $J-H$ & $J-K$ \\
\hline 13 June 06:00 & $16.10 \pm 0.02$ & $0.56 \pm 0.03$ & $0.33 \pm 0.03$ & $0.67 \pm 0.03$ & 13 June 02:35 & $15.11 \pm 0.04$ & $0.28 \pm 0.06$ & $0.35 \pm 0.06$ \\
13 June 07:08 & $16.14 \pm 0.02$ & $0.60 \pm 0.03$ & $0.34 \pm 0.03$ & $0.63 \pm 0.04$ & 13 June 06:15 & $15.15 \pm 0.03$ & $0.30 \pm 0.06$ & $0.36 \pm 0.06$ \\
13 June 08:46 & $16.05 \pm 0.02$ & $0.64 \pm 0.03$ & $0.28 \pm 0.04$ & $0.63 \pm 0.03$ & 14 June 02:38 & $15.08 \pm 0.03$ & $0.27 \pm 0.05$ & $0.32 \pm 0.04$ \\
mean & $16.10 \pm 0.02$ & $0.60 \pm 0.03$ & $0.32 \pm 0.03$ & $0.64 \pm 0.03$ & mean & $15.10 \pm 0.03$ & $0.28 \pm 0.06$ & $0.34 \pm 0.05$ \\
\hline
\end{tabular}

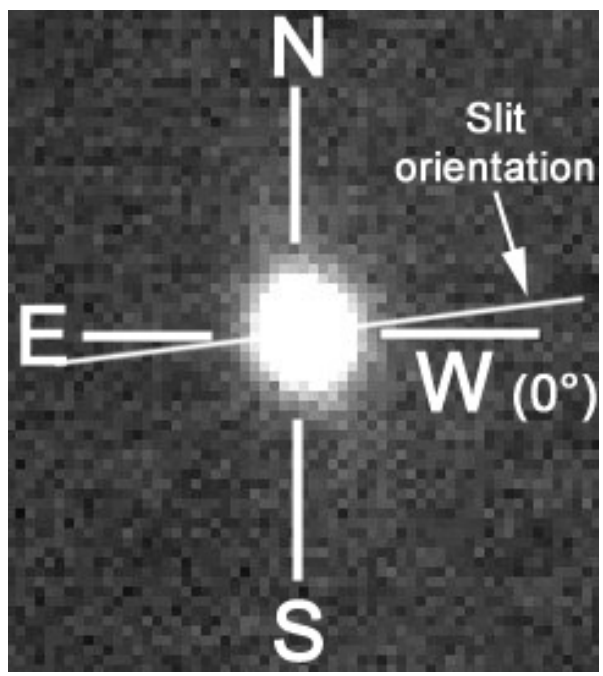

Fig. 1. $V_{1}$ combined frame. Orientation conventions, and slit orientation are superimposed on the frame. Since the phase angle is small, the Sun direction is roughly aligned with the observer direction. The slit is oriented along the object's motion. This extracted frame is $12^{\prime \prime}$ wide.

diverges from the stellar profile, showing evidence for the presence of a resolved coma. On combined frames, a slight asymmetric shape of the coma could be distinguished (see Fig. 1). Thus, in order to investigate this asymmetry, we have also computed the Chiron profile in a $90^{\circ}$ quadrant, centred on four directions: North $\left(90^{\circ}\right)$, East $\left(180^{\circ}\right)$, South $\left(-90^{\circ}\right)$, and West $\left(0^{\circ}\right)$.

Figure 2 shows the profiles computed from the $V_{1}$ frame, integrated on $90^{\circ}$ centred on four preferred directions (NESW). Figure 1 indicates the angular conventions, superimposed on the $V_{1}$ combined frame. The signal from Chiron's coma is stronger on frames (1) and (3). It is lower on frame (4), accounting for the bump around 4" which is due to bad star subtraction. The signal from Chiron's coma is below the noise level in frame (2). Profiles computed from frames $V_{2}$ and $V_{3}$ give similar results, but with lower signal-to-noise ratio due to larger seeing. The signal from the coma is above noise level up to $6^{\prime \prime}-$ radius.

The shape of the coma appears to be slightly asymmetric: the coma is roughly oriented along the North-South direction. Luu \& Jewitt (1990) detected an elongated coma in the antisolar direction, and suggested that it was due to radiation pressure effects on micron-sized grains. Meech \& Belton (1990) also noticed an extended coma in the antisolar direction. However, Fulle (1994) suggested that the asymmetry of the coma could be explained by nucleus rotation. Within our work, the phase

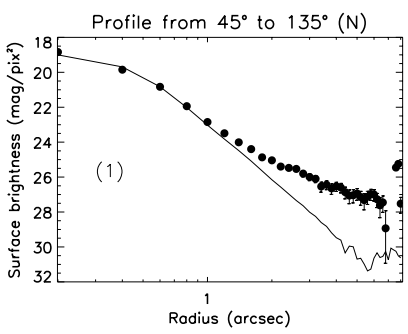

Profile from $225^{\circ}$ to $315^{\circ}$ (S)

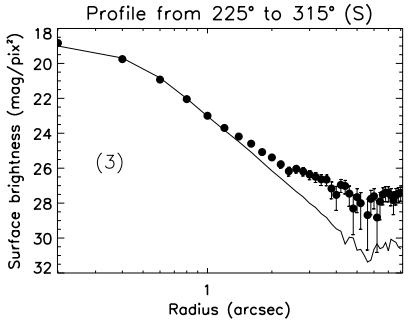

Fig. 2. Surface brightness profiles, computed from the $V_{1}$ frame. Each profile has been computed between two angular positions indicated on top of each frame. The angular conventions are reported on Fig. 1. Both Chiron (dots) and stellar (continuous line) profiles extend to $8^{\prime \prime}$. Beyond $6-7$ ", however, Chiron's profile becomes very noisy.

angle is very small $\left(0.7^{\circ}\right)$, so that it is difficult to detect any elongation of the coma in the solar/antisolar direction.

Using a least-square method, we have computed the slope of the surface brightness profile defined by $s=\mathrm{d} B / \mathrm{d} \log p$ were $B$ is the surface brightness in flux $/ \operatorname{arcsec}^{2}$ and $p$ is the projected distance from the nucleus (in $\operatorname{arcsec}$ ). Within the $1.8-6.0^{\prime \prime}$ range, we found $s \simeq-2$, which is smaller than expected from a steady-state coma $(-1 \geq s \geq-1.5$, Jewitt \& Meech 1987). Such steep profiles have already been noticed by several authors (Luu \& Jewitt 1990; Meech \& Belton 1990), who have suggested that they are due to outburst activity. This is only a rough determination of the slope $s$, since we have measured the surface brightness profile within a small extension range.

\subsection{Dust production rate}

In order to compare dust production rates from different comets, A'Hearn et al. (1984) introduced the Af $\rho$ parameter (in $\mathrm{cm}$ ), which is defined as:

$A f \rho=\left(\frac{2 \Delta R_{\mathrm{h}}}{\rho}\right)^{2} 10^{-0.4\left(m_{\mathrm{coma}}-m_{\mathrm{sun}}\right)}$

where $A$ is the albedo, $f$ is the filling factor of grains within the chosen aperture, and $\rho$ is the projected aperture size (in $\mathrm{cm}$ ) 
used to measure the coma flux. $\Delta$ is the geocentric distance (in $\mathrm{cm}$ ), $R_{\mathrm{h}}$ is the heliocentric distance (in $\mathrm{AU}$ ), $m_{\text {coma }}$ is the magnitude of the coma, and $m_{\text {sun }}$ is the solar magnitude at $1 \mathrm{AU}$.

We have measured the flux from the coma by extrapolating the surface brightness profile of the coma through the nucleus vicinity. We have used two different values for the slope $s$ ( $s=$ -1.5 and $s=-2.5$ ) since our estimate of the slope is very approximate. We have then computed an estimate of $A f \rho$, that would range between $3600 \mathrm{~cm}$ (for $s=-1.5$ ) and $12000 \mathrm{~cm}$ (for $s=-2.5$ ).

Measurements of Af $\rho$ have been obtained for Schwassmann-Wachmann $1(2900 \mathrm{~cm}$ at $6.2 \mathrm{AU}$, Jorda 1995), and Hale-Bopp (50000 $\mathrm{cm}$ at $7 \mathrm{AU}$, Schleicher et al. 1997). Activity in distant comets is expected to be mainly driven by $\mathrm{CO}$ sublimation, whereas other volatile compounds (e.g. $\mathrm{N}_{2}$ ) could also be present (see discussion in Bus et al. 2001). Biver et al. (2002) found that the CO production rate in Hale-Bopp was proportional to $R_{h}^{-2}$. In order to compare Chiron to SW1 and Hale-Bopp, we assumed that the dust production rate has the same behaviour as the one for $\mathrm{CO}$, and estimated $A f \rho$ at $1 \mathrm{AU}$. We obtained $4 \times 10^{5}-1.4 \times 10^{6} \mathrm{~cm}$, $1.1 \times 10^{5} \mathrm{~cm}$, and $2 \times 10^{6} \mathrm{~cm}$, for Chiron, SW1, and Hale-Bopp respectively.

Af $\rho$ is directly related to the dust production rate by:

$\frac{\mathrm{d} \mathcal{M}}{\mathrm{d} t}=C \times A f \rho$

where $C$ is a function of the grain size distribution, the dust velocity, the grain density, the geometric albedo of the grain, and a phase factor (Jorda 1995). We have neglected grain size distribution, and assumed that the mean grain size is $1 \mu \mathrm{m}$ (small grains of $1-\mu \mathrm{m}$ size strongly contribute to the flux at $500 \mathrm{~nm}$ ). We have used $15 \mathrm{~m} \mathrm{~s}^{-1}$ for the dust grain velocity, following modelling attempts by Fulle (1994 and personal communication). We have also assumed a geometric albedo of 0.04 , and a phase factor of 1 (since the observations were performed at small phase angle, and Chiron is a distant target). The dust production rate would be included in the range $7-23 \mathrm{~kg} \mathrm{~s}^{-1}$.

\subsection{Chiron spectra}

The spectroscopic data reduction technique has been described in Sect. 2. In order to increase the signal-to-noise ratio, the spectral resolution has been degraded to 150 in the optical range and 250 in the NIR range. The signal-to-noise ratio of the final spectra ranges between 70 and 100 depending on the wavelength. Optical spectra are shown in Fig. 3 and they do not show any significant features. We checked that the increase of the slope beyond $0.8 \mu \mathrm{m}$ is not due to airmass effects or instrumental effects. Since there is no other spectrum of Chiron published at these wavelengths, we cannot check the validity of this spectral behaviour.

NIR spectra are shown in Fig. 4. There are no obvious water ice absorption features. The three $J, S H$, and $S K$ segments are adjusted together using the average $J-H$ and $J-K$ colors. Slight changes appear in the $K$ spectra. However, considering the noise level and the rotational phase of each spectrum (using

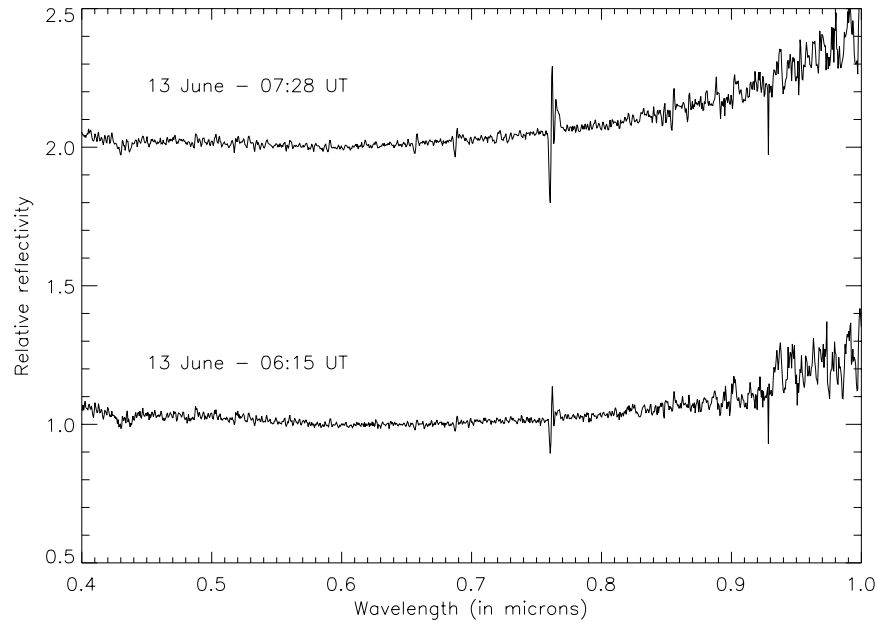

Fig. 3. Optical spectra of Chiron. The spectra have been normalized at $0.55 \mu \mathrm{m}$, and the upper spectrum has been shifted by 1 for clarity. The feature around $0.76 \mu \mathrm{m}$ is due to bad correction of atmospheric features.

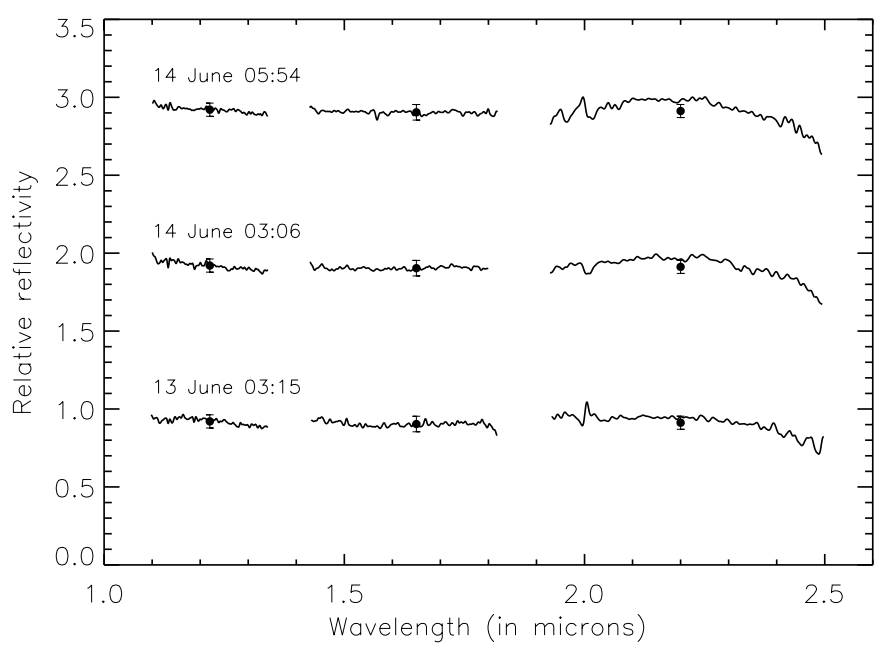

Fig. 4. Near-infrared spectra of Chiron. The upper spectra have been shifted for clarity. Features around $2 \mu \mathrm{m}$ are due to bad correction of atmospheric features.

the lightcurve from Marcialis \& Buratti 1993), these changes would not be significant. An absorption at wavelengths higher or equal to $2.25 \mu \mathrm{m}$ is present in all three spectra. Comparable absorptions have been detected in spectra of other outer solar system objects: Hale-Bopp when it was still at 7 AU from the Sun (Davies et al. 1997), other Centaurs and some TNOs (M.E. Brown, unpublished data), but no identification of the component(s) responsible for this absorption has ever been made.

We could not combine the visible part with the NIR part of Chiron's spectrum, since the contribution of the coma in the visible range is stronger than in the NIR range (for dust grains of $\simeq 1 \mu \mathrm{m}$ ). The contribution of the coma in the spectrum is also different from the one in magnitude measurements, mainly because the slit is oriented perpendicularly to the preferred direction of the coma (Fig. 1). Thus the visible and NIR parts of the spectrum cannot be adjusted together due to two main reasons: (i) the absolute calibration of the spectrum using the 
magnitude measurements would not be correct, and (ii) a combined Visible + NIR spectrum is meaningless since the contribution of the coma is stronger in the visible part than in the NIR part.

\section{Conclusion}

The major results of this work are that (i) an activity outburst occurred at Chiron around 13 June 2001, (ii) no water ice absorption feature could be seen in NIR spectra. The lack of water ice absorption bands together with the high activity level of Chiron confirm the hypothesis made by Luu et al. (2000): the detection of water ice in Chiron spectra would be correlated with its cometary activity level.

The absolute magnitude measurement shows that Chiron was at a high activity level in June 2001, that had not been reached for 10 years. Accounting for the estimate by Bauer et al. (2001), Chiron could even have been as active as in 1988 .

Acknowledgements. We would like to thank Dominique BockeléeMorvan and Olivier Hainaut for providing helpful comments on this work. Support was provided to C. Delahodde by the Société de Secours des Amis des Sciences.

\section{References}

A'Hearn, M. F., Schleicher, D. G., Millis, R. L., et al. 1984, AJ, 89, 579

Barucci, M. A., Romon, J., Doressoundiram, A., et al. 2000, AJ, 120, 496

Barucci, M. A., Cruikshank, D. P., Mottola, S., et al. 2002, in Asteroids III, in press
Bauer, J. M., Meech, K. J., Owen, T. C., et al. 2001, BAAS, 33, 12.12 Biver, N., Bockelée-Morvan, D., Colom, P., et al. 2002, in Cometary Science After Hale-Bopp, IAU Colloq. 186, Tenerife, Spain, in press

Bus, S. J., Bowell, E., \& Harris, A. W. 1989, Icarus, 77, 223

Bus, S. J., A'Hearn, M. F., Schleicher, D. G., et al. 1991, Science, 251, 774

Bus, S. J., A'Hearn, M. F., \& Bowell, E. 2001, Icarus, 150, 94

Campins, H., Telesco, C. M., Osip, D. J., et al. 1994, AJ, 108, 2318

Davies, J. K., Roush, T. L., Cruikshank, D. P., et al. 1997, Icarus, 127, 238

Davies, J. K., McBride, N., \& Ellison, S. L. 1998, Icarus, 134, 213

Fernández, Y. R., Jewitt, D. C., \& Sheppard, S. S. 2002, AJ, 123, 1050

Foster, M. J., Green, S. F., McBride, N., et al. 1999, Icarus, 141, 408 Fulle, M. 1994, A\&A, 282, 980

Groussin, O., Lamy, P., Peschke, S., et al. 2002, Icarus, in press

Hartmann, W. K., Tholen, D. J., Meech, K. J., et al. 1990, Icarus, 83, 1

Jewitt, D. C., \& Meech, K. J. 1987, ApJ, 317, 992

Jorda, L. 1995, Ph.D. Thesis

Kowal, C. T., Liller, W., \& Chaisson, L. J. 1977, IAU Circ., 3147, 1

Landolt, A. U. 1992, AJ, 104, 340

Lazzaro, D., Florczak, M. A., Angeli, C. A., et al. 1997, Planet. Space Sci., 45, 1607

Levison, H. F., \& Duncan, M. J. 1997, Icarus, 127, 13

Luu, J. X., \& Jewitt, D. C. 1990, AJ, 100, 913

Luu, J. X., Jewitt, D. C., \& Trujillo, C. 2000, ApJ, 531, L151

Marcialis, R. L., \& Buratti, B. J. 1993, Icarus, 104, 234

Meech, K. J., \& Belton, M. J. S. 1989, IAU Circ., 4770, 1

Meech, K. J., \& Belton, M. J. S. 1990, AJ, 100, 1323

Romon, J., de Bergh, C., Barucci, M. A., et al. 2001, A\&A, 376, 310

Schleicher, D. G., Lederer, S. M., Millis, R. L. 1997, Science, 275, 1913

Tholen, D. J., Hartmann, W. K., Cruikshank, D. P., et al. 1988, IAU Circ., 4554, 2

Womack, M. \& Stern, S. A. 1995, IAU Circ., 6193, 1 